\title{
Overexpression of Transient Receptor Protein Cation Channel Subfamily A Member 1, Confers an Independent Prognostic Indicator in Nasopharyngeal Carcinoma
}

\author{
You-Ting $\mathrm{Wu}^{1,2^{*}}$, Shao-Lun Yen ${ }^{*}$, Chien-Feng $\mathrm{Li}^{3,4,5,6}$, Ti-Chun Chan ${ }^{3}$, Tzu-Ju Chen ${ }^{3}$, Sung-Wei Lee7, \\ Hong-Lin $\mathrm{He}^{8,9}$, I-Wei Chang8, Chung-Hsi Hsing10, Yow-Ling Shiue 9,11,12区 \\ 1. Departments of Pathology, Kaohsiung Chang Gung Memorial Hospital and Chang Gung University College of Medicine, Kaohsiung, Taiwan; \\ 2. Department of Pathology, Chang Gung Memorial Hospital, Chia-Yi, Taiwan; \\ 3. Department of Pathology, Chi-Mei Medical Center, Tainan, Taiwan; \\ 4. National Institute of Cancer Research, National Health Research Institutes, Tainan, Taiwan; \\ 5. Department of Biotechnology, Southern Taiwan University of Science and Technology, Tainan, Taiwan; \\ 6. Institute of Clinical Medicine, Kaohsiung Medical University, Kaohsiung, Taiwan; \\ 7. Department of Radiation Oncology, Chi-Mei Medical Center, Liouying, Tainan, Taiwan; \\ 8. Department of Pathology, E-DA Hospital, I-Shou University, Kaohsiung, Taiwan; \\ 9. Institute of Biomedical Sciences, National Sun Yat-sen University, Kaohsiung, Taiwan; \\ 10. Department of Anesthesiology, Kaohsiung Chang Gung Memorial Hospital and Chang Gung University College of Medicine, Kaohsiung, Taiwan; \\ 11. Department of Biological Sciences, National Sun Yat-sen University, Kaohsiung, Taiwan; \\ 12. Doctoral degree program in Marine Biotechnology, National Sun Yat-sen University, Kaohsiung, Taiwan.
}

*These authors contributed equally to this work.

$\square$ Corresponding author: Yow-Ling Shiue, PhD. Institute of Biomedical Sciences, National Sun Yat-sen University, 70 Lienhai Rd. 80424 Kaohsiung, Taiwan. Email: ylshiue@mail.nsysu.edu.tw Ph: 886-7-5255818 Fax: 886-7-5250197.

() Ivyspring International Publisher. Reproduction is permitted for personal, noncommercial use, provided that the article is in whole, unmodified, and properly cited. See http://ivyspring.com/terms for terms and conditions.

Received: 2016.02.19; Accepted: 2016.04.26; Published: 2016.06.08

\begin{abstract}
Background: Detection of oncogenes provides chances to understand tumor development and progression. Transient receptor protein cation channel subfamily A, member 1 (TRPAI) transcript was significantly upregulated in nasopharyngeal carcinoma (NPC) with a stepwise upregulation from low- to high-stage NPCs from a preliminary data analysis in the Gene Expression Omnibus database. The TRPA I gene is a member of the TRP channel family, encoding integral membrane proteins that functions as cation channels. Loss of calcium homeostasis takes place in cancer cells.

Methods: Immunostaining of TRPA1 was analyzed on 124 biopsies from NPC patients retrospectively. The $\mathrm{H}$-score method was used to evaluate the immunoexpression of TRPAl. The correlations between $\mathrm{H}$-score of TRPAl protein level and clinicopathological factors, as well as the significances of TRPAl protein level for disease-specific, distal-metastasis-free and local recurrence-free survivals were assessed.

Results: These patients were characterized to be no initial metastasis and medicated with the traditional procedure. The TRPAl score was found to be associated with clinicopathological parameters and patient survivals. Along with the guideline of 7 th edition of the American Joint Committee on Cancer, we found that TRPAl upregulation (50\%) was associated with advanced primary tumor $(P=0.009)$ and overall clinical stage $(P=0.019)$. In univariate log-rank testing, primary tumor, nodal status, stage and TRPAI protein level significantly contributed to worse disease-specific survival, distal metastasis-free survival and local recurrence-free survival. In multivariate analysis, high TRPAI protein level and tumor stage emerged as independent prognostic indicators for inferior disease-specific survival $(P=0.014 ; P=0.003)$, distal metastasis-free survival $(P=0.004 ; P=0.034)$ and recurrence-free survival $(P=0.017 ; P=0.015)$.
\end{abstract}


Conclusions: The upregulation of TRPAI protein level is frequently correlated to unfavorable prognosticators and gives rise to cancer progression in NPC patients.

Key words: nasopharyngeal carcinoma, TRPA1, prognosis.

\section{Introduction}

Both environmental and genetic components are responsible for the pathogenesis of nasopharyngeal carcinoma (NPC) [1]. Epstein-Barr virus (EBV) infection is related to the development of NPC, a prevalent head and neck tumor in Taiwan and southern Asia [2]. On the basis of modern World Health Organization (WHO) tumor categorization, the correlation is high and predominant in differentiated as well as undifferentiated non-keratinizing carcinomas [3]. In NPC patients, recent progressions have been made in imaging diagnosis, radiation treatment, adjuvant chemotherapy and neoadjuvant chemotherapy. These treatments enhance locoregional cure, nevertheless, therapeutic alternatives for progressive NPCs are still insufficient [4], although the additional neoadjuvant chemotherapy plus concurrent chemoradiotherapy is associated with reduced distant failure as compared with concurrent chemoradiotherapy alone [5]. Moreover, chemoradiotherapy-induced hemoglobin decrease has negative influence on locoregional control and survival [6]. In keeping with the American Joint Committee on Cancer (AJCC, $7^{\text {th }}$ edition) stratification, primary tumor, nodal status and metastasis continue to be critical prognostic elements [3,4]. Thus, to detect potential biomarkers with improved associations to malignancy development and/or medication results in NPC patients are indispensable. Novel markers will be helpful to classify and explore novel therapeutic targets for NPC [7].

Data mining on a generally-acknowledged expression profile in the Gene Expression Omnibus dataset (GEO, NCBI, Bethesda, MD, USA) was accordingly performed. Among three potential transcripts that were considerably higher expressed in human NPCs, transient receptor potential cation channel, subfamily A, member 1 (TRPA1) presented the highest $\log _{2}$ ratio $(P<0.0001)$ and a correlation coefficient with the tumor stage $(P=0.0002)$. The TRPA1 gene belongs to the TRP channel family, encoding integral membrane proteins that functions as cation channels. So far, about 30 TRPs have been recognized. Through protein homology and channel function, these TRPs are characterized into seven distinct subfamilies: TRPC (canonical), TRPV (vanilloid), TRPM (melastatin), TRPML (mucolipin), TRPP (polycystin), TRPA (ankyrin transmembrane protein) and, TRPN (NomPC-like). Genes encoding TRP ion channels are broadly expressed in various tissues and cell categories [8]. These genes participate in distinctive physiological processes, for example, the perception of diverse stimuli or ion homeostasis [9, 10].

Except for a few are high $\mathrm{Ca}^{2+}$-selective and some are even permeable, for greatly hydrated $\mathrm{Mg}^{2+}$ ions, most TRPs are non-selective cation channels. A standard TRP protein consists of six putative transmembrane $\alpha$-helices (S1 to S6) and surrounded by cytoplasmic $\mathrm{N}$ and $\mathrm{C}$ terminals, with a pore-shaping reentrant loop between S5 and S6 [11-13]. Lately, TRP channels were reported to engage in the activation of cell proliferation and abnormal differentiation, as well as resistance to apoptosis. These aspects result in tumor growth and progression [14]. Numerous TRP channel genes, TRPC1, TRPC3, TRPC4, TRPC5, TRPC6, TRPM2, TRPM4, TRPM7, TRPM8, TRPV1, TRPV2, TRPV4 and TRPV6, have been reported to play oncogenic roles in cancer cells, including the carcinoma of lung, breast, prostate, ovarian, gastric, liver, nasopharyngeal, glioblastoma and melanoma [15]. Yet, the immunoexpression of TRPA1 in a considerable set of biopsies from NPC tissues and its correlation with various clinicopathological parameters have not been carefully evaluated. Hence, the aim of this investigation was to inspect their relationships.

\section{Materials and methods}

\section{Analysis of expression profile}

Firstly, we identified a database (http://www.ncbi.nlm.nih.gov/geo/query/acc.cgi?a $\mathrm{cc}=\mathrm{GSE} 12452)$, containing the results of 31 biopsies of NPCs along with 10 health nasopharyngeal tissues which were analyzed by the Human Genome U133 Plus 2.0 Array (Affymetrix, Santa Clara, CA, USA). To calculate the expression values of all transcripts, original CEL files were introduced into the Nexus Expression 3 software (BioDiscovery, EI Segundo, CA, USA). Devoid of prior selection and filtering, the entire probe series were utilized in the computation. Based on tissue types [NPCs vs. non-tumor tissues; high-stage (stage III) vs. low-stage (stage I-II) NPCs], comparative analysis with supervision was executed, to determine the significantly and differentially 
expressed genes, i.e., critical transcripts in the pathogenesis and progression of NPC. Those transcripts with $P \leq 0.005$ and $\log _{2}$-transformed expression fold-change $>1$ are listed in Table 1 .

\section{Patients and tumor samples}

Tissues from NPC patients were formalin-fixed, permitted by the institutional review board and used in this survey (IRB1030213). Paraffin-embedded tissues were created from 124 NPC patients. Biopsies of these patients were undertaken during January 1993 to December 2002. Distant metastasis was not shown in any individual during initial presentation. The tumor staging and histological subtypes were reassessed according to the $7^{\text {th }}$ AJCC procedure and the current $\mathrm{WHO}$ classification by two pathologists, objectively.

\section{Immunohistochemistry}

From paraffin-embedded tissue blocks, tissue sections (3- $\mu \mathrm{m}$ thickness) were cut onto precoated slides. Xylene and ethanol were used to deparaffinize and rehydrate the slides. To retrieve antigens, slides were microwaved in the 10-mM citrate buffer ( $\mathrm{pH}$ 6.0; $7 \mathrm{~min}$ ). Saline was used to buffer endogenous peroxidase. Slides were then developed using a goat polyclonal primary antibody probing TRPA1 (sc-32353, Santa Cruz Biotechnology, Santa Cruz, CA, USA) at a dilution ratio of 1:25 (1 h). The ChemMate ${ }^{\mathrm{TM}}$ DAKO EnVision ${ }^{\mathrm{TM}}$ Detection Kit (K5001, Carpinteria, CA, USA) was applied to detect the primary antibody. The slides were next treated with an anti-goat secondary antibody for $30 \mathrm{~min}$ and 3,3-diaminobenzidine for $5 \mathrm{~min}$. Thereafter, Gill's haematoxylin was applied for counterstaining. Without knowing any clinical and follow-up data, the immunostaining of the TRPA1 protein were examined blindly by two pathologists (CF Li and TJ Chen). In the cytoplasm and cell membrane of the tumor cells, immunoreactivities were assessed with the joint of the intensity and percentage of positively stained tumor cells. To produce the $\mathrm{H}$-score, the equation, $\mathrm{H}$-score $=$
$\Sigma P_{i}(i+1)$, was used. $P i$ stands for the percentage while $i$ is the intensity of cancer cells stained by the anti-TRPA1 antibody (0 to $3+)$. The intensity varied from 0 to $100 \%$. H-score > median of total specimens were designated as high TRPA1 expression level.

\section{Patient medication and clinical characteristics}

Every patient with follow-up for consequence accepted an entire program of radiotherapy (total dose $\geq 7,000$ cGy). In patients with stages II-IV, cisplatin-based chemotherapy was additionally treated according to the former procedure [14]. The process of radiotherapy was overall constant during this time slot. Each patient was consistently censored following radiation therapy till expiration or his/her final appointment. The average follow-up interval was 59.6 months, spanning 4-117 months.

\section{Statistics}

The SPSS V.14.0 software (SPSS Inc. Chicago, IL, USA) was used to conduct statistical analyses. To test the independence between TRPA1 protein expression level and different clinicopathological parameters, Chi-square test was applied. Disease-specific survival, distal metastasis-free survival, and local recurrence-free survival are the endpoints that were analyzed. These were determined from the beginning day of radiation therapy, to the day of event occurred. Cases without patient clinical characteristics were inspected at the final day of follow-up. To plot survival curves and estimate the prognostic variations between patient clusters, Kaplan-Meier technique and the log-rank test were used. With the Cox proportional hazard model, multivariate analysis was carried out. Nevertheless, primary tumor and nodal status (both are AJCC components) were not incorporated in multivariate estimations. Two-tailed analyses of significance were employed and $P<0.05$ was regarded as statistical significance for all calculations.

Table 1. Summary of top 3 differentially expressed transcripts associated with both initiation and progression of NPC in the transcriptome of nasopharyngeal carcinoma.

\begin{tabular}{|c|c|c|c|c|c|c|c|}
\hline \multirow{2}{*}{$\begin{array}{l}\text { Gene } \\
\text { Symbol }\end{array}$} & \multirow[t]{2}{*}{ Probe } & \multicolumn{2}{|c|}{ Comparing tumor to non-tumor } & \multicolumn{2}{|c|}{ Comparing high to low stage \& } & \multirow[t]{2}{*}{ Gene Name } & \multirow[t]{2}{*}{ Molecular Function } \\
\hline & & $\begin{array}{l}\text { Comparison } \\
\log \text { ratio }\end{array}$ & $\begin{array}{l}\text { Comparison } \\
P \text {-value }\end{array}$ & $\begin{array}{l}\text { Comparison } \\
\text { log ratio }\end{array}$ & $\begin{array}{l}\text { Comparison } \\
P \text {-value }\end{array}$ & & \\
\hline TRPA1 & 217590_s-at & 1.3009 & 0.0003 & 2.0258 & $<0.0001$ & $\begin{array}{l}\text { Transient receptor potential } \\
\text { cation channel; subfamily A; } \\
\text { member } 1\end{array}$ & $\begin{array}{l}\text { Calcium channel activity, calcium } \\
\text { ion binding, channel activity, ion } \\
\text { channel activity }\end{array}$ \\
\hline \multirow[t]{2}{*}{ C8orf42 } & 226778_at & 1.2737 & $<0.0001$ & 1.1512 & 0.0002 & \multirow{2}{*}{$\begin{array}{l}\text { Chromosome } 8 \text { open reading } \\
\text { frame } 42\end{array}$} & \multirow[t]{2}{*}{-} \\
\hline & 230903_s_at & 1.1358 & $<0.0001$ & 1.047 & 0.0005 & & \\
\hline \multirow[t]{2}{*}{ CALB1 } & 205626_s_at & 1.1786 & 0.0013 & 1.1036 & 0.0066 & \multirow[t]{2}{*}{ Calbindin $1 ; 28 \mathrm{kDa}$} & \multirow{2}{*}{$\begin{array}{l}\text { Calcium ion binding, protein } \\
\text { binding, vitamin } \mathrm{D} \text { binding }\end{array}$} \\
\hline & 205625_s_at & 1.0469 & 0.0016 & 1.0743 & 0.0027 & & \\
\hline
\end{tabular}

$\&$, high stage: stage III; low stage: stage I and II. 


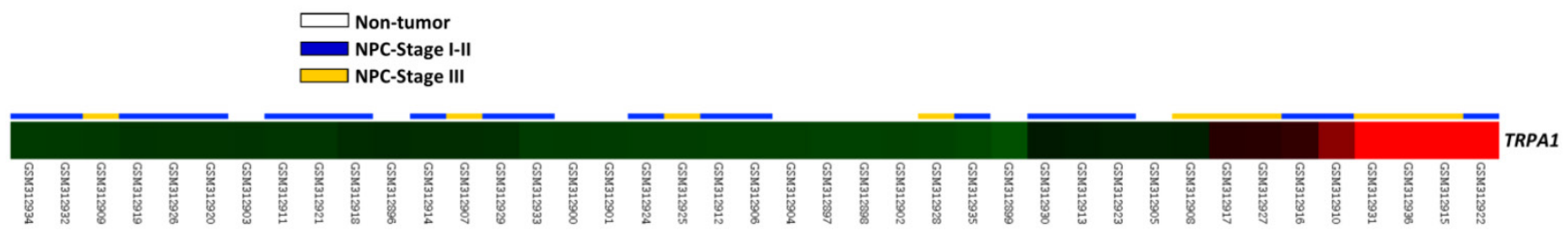

Figure 1. A heat map showed that data analysis on GSE12452 (GEO dataset) detected TRPAI transcript constantly upregulated $(P<0.0001)$ in tissues from NPC, compared to those from normal ones, and TRPAI transcript showed a stepwise upregulation in low- to high-stage NPCs. Sample identities of normal $(n=10)$ and tumor $(n=31)$ are indicated. Green, dark and red represent low, intermediate and high expression values.
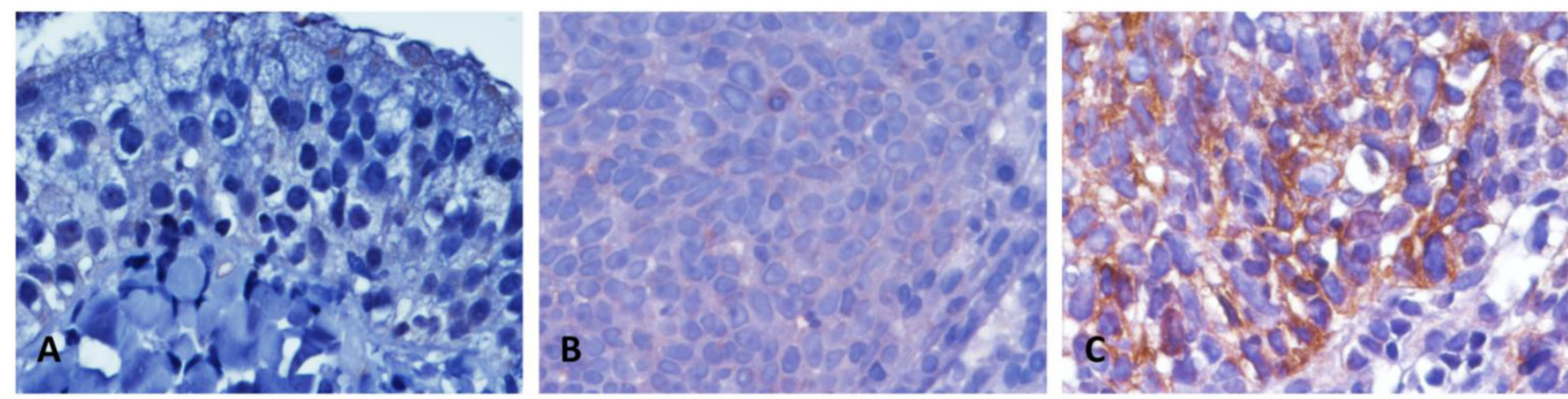

Figure 2. Representatives of the TRPA1 immunoexpression in normal and tumor tissues of NPC patients. In a normal nasopharyngeal mucosa, TRPA1 was not detected (A), (B) NPC tissues with low expression (H-score $\leq$ median) and (C) high TRPAl staining (H-score > median).

\section{Results}

\section{The TRPAI transcript was considerably upregulated in NPCs deposited in the public database}

From GSE12452 deposited in GEO database containing 10 normal and 31 NPC tissues, we found that five probes representing three transcripts were significantly upregulated in tissues from NPC patients, compared to those from normal tissues $(P<$ 0.0001; Table 1). Of these transcripts, the expression level of TRPA1 mRNA also revealed a stepwise upregulation in low- to high-stage NPCs $(P=0.0002$; Fig. 1). Therefore, immunoexpression and clinical significances of TRPA1 in a larger cohort was further studied.

\section{Immunoexpression of TRPA1 and its correlation with clinicopathological parameters in NPC specimens}

Thoroughly, 124 patients with NPC containing five keratinizing squamous cell, 54 non-keratinizing differentiated, and 65 non-keratinizing, undifferentiated carcinomas were studied. With an average age of 48.6 years (ranging from 20 to 83 years), this cohort contains 95 males and 29 females. Thirty-eight patients were categorized into AJCC stages I-II; 86 were stages III-IV (Table 2). Immunoexpression of the TRPA1 protein was distinctly examined in each specimen. The TRPA1 protein was barely detected in a normal nasopharyngeal mucosa (Fig. 2A). Due to the H-score of 62 patients were examined to be lower than the median value, these events were classified as TRPA1 low expression (Fig. 2B). Additional 62 specimens showed distinguishing TRPA1 immunostaining, with the $\mathrm{H}$-score higher than the median value (Fig. 2C). As shown in Table 2, high TRPA1 protein levels were significantly correlated to those specimens with advanced primary tumor (T3-T4; $P=0.009$ ) and higher AJCC stage $(P=0.019)$. In contrast, other clinicopathological parameters were not meaningfully correlated with the TRPA1 protein level (Table 2).

Table 2. Correlation between TRPAl expression levels and important clinicopathologic variables.

\begin{tabular}{|c|c|c|c|c|}
\hline \multirow[t]{2}{*}{ Parameter } & \multirow[t]{2}{*}{ Category } & \multicolumn{2}{|c|}{ TRPA1 expression level } & \multirow[t]{2}{*}{$P$-value } \\
\hline & & Low & High & \\
\hline \multicolumn{5}{|l|}{ Gender } \\
\hline & Male & 48 & 47 & 0.832 \\
\hline & Female & 14 & 15 & \\
\hline \multicolumn{5}{|l|}{ Age (years) } \\
\hline & $<60$ & 47 & 51 & 0.378 \\
\hline & $\geq 60$ & 15 & 11 & \\
\hline \multicolumn{5}{|c|}{ Primary tumor $(\mathrm{T})$} \\
\hline & $\mathrm{T} 1-\mathrm{T} 2$ & 47 & 33 & $0.009^{*}$ \\
\hline & $\mathrm{T} 3-\mathrm{T} 4$ & 15 & 29 & \\
\hline \multicolumn{5}{|c|}{ Nodal status (N) } \\
\hline & N0-N1 & 33 & 23 & 0.071 \\
\hline & N2-N3 & 29 & 39 & \\
\hline \multicolumn{5}{|l|}{ Stage } \\
\hline & I-II & 25 & 13 & $0.019^{*}$ \\
\hline & III-IV & 37 & 49 & \\
\hline \multicolumn{5}{|c|}{ Histological grade } \\
\hline & Keratinizing & 3 & 2 & 0.445 \\
\hline & Non-keratinizing & 30 & 24 & \\
\hline & Undifferentiated & 29 & 36 & \\
\hline
\end{tabular}




\section{The prognostic effect of TRPAl protein levels on NPCs}

As shown in Table 3, NPC patients with lower primary tumor (T1-T2), nodal status (N0-N1) and AJCC stage (I-II) ensured better disease-specific survival $(P=0.0289 ; P=0.0008 ; P=0.0020)$, distal metastasis-free survival $(P=0.0085 ; P=0.0132 ; P=$ $0.0072)$ and local recurrence-free survival $(P=0.0180$; $P=0.0160 ; P=0.0026)$, within a medium period of 64 months. Moreover, high TRPA1 protein levels were convincingly prognostic of disease-specific survival $(P$ $=0.003)$, distal metastasis-free survival $(P=0.0005)$ and local recurrence-free survival $(P=0.0026)$ (Fig 3). Next to AJCC tumor stage [16], a high TRPA1 protein level steadily served as a negative prognostic indicator for disease-specific survival $(P=0.014$; hazard ratio $=2.297)$, distal metastasis-free survival $(P$ $=0.004$; hazard ratio $=2.471)$ and local recurrence-free survival $(P=0.017$; hazard ratio $=2.335)$ in multivariate analysis (Table 4). Therefore, the protein level of TRPA1 represents as an independent prognostic marker.

\section{Discussion}

In the current report, we showed that upregulation of TRPA1 protein can be a strong prognostic marker for disease-free, distal metastasis-free and local recurrence-free survivals in a cohort of 124 NPC patients. As a matter of fact, the expression patterns of other TRP channel members have been suggested to be biomarkers for diagnosis and/or prognostics in different cancers. For instance, high TRPC6 expression in esophageal squamous cell carcinoma was reported, and it is essential for cell propagation and the progression of cell cycle [17]. In metastatic breast cancer, TRPV6 and TRPM7 may act as biomarkers by correlation with detrimental outcome [18].

Table 3. Univariate log-rank analysis.

\begin{tabular}{|c|c|c|c|c|c|c|c|c|}
\hline \multirow{2}{*}{$\begin{array}{l}\text { Parameter } \\
\mathrm{s}\end{array}$} & \multirow[t]{2}{*}{ Category } & \multirow[t]{2}{*}{$n$} & \multicolumn{2}{|c|}{ DSS } & \multicolumn{2}{|c|}{ DMeFS } & \multicolumn{2}{|c|}{ LRFS } \\
\hline & & & $n$ & $P$-value & $n$ & $P$-value & $n$ & $P$-value \\
\hline \multicolumn{9}{|l|}{ Gender } \\
\hline & Male & 95 & 45 & 0.7870 & 38 & 0.6128 & 30 & 0.3240 \\
\hline & Female & 29 & 14 & & 11 & & 7 & \\
\hline \multicolumn{9}{|l|}{$\begin{array}{l}\text { Age } \\
\text { (years) }\end{array}$} \\
\hline & $<60$ & 98 & 48 & 0.8600 & 42 & 0.3091 & 29 & 0.8206 \\
\hline & $\geq 60$ & 26 & 11 & & 7 & & 8 & \\
\hline \multicolumn{9}{|l|}{$\begin{array}{l}\text { Primary } \\
\text { tumor }(\mathrm{T})\end{array}$} \\
\hline & $\mathrm{T} 1-\mathrm{T} 2$ & 80 & 32 & $0.0289^{*}$ & 25 & $0.0085^{*}$ & 19 & $0.0180^{*}$ \\
\hline & T3-T4 & 44 & 27 & & 24 & & 18 & \\
\hline \multicolumn{9}{|l|}{$\begin{array}{l}\text { Nodal } \\
\text { status }(\mathrm{N})\end{array}$} \\
\hline & N0-N1 & 56 & 18 & $0.0008^{*}$ & 17 & $0.0132^{*}$ & 12 & $0.0160^{*}$ \\
\hline & N2-N3 & 68 & 41 & & 32 & & 25 & \\
\hline \multicolumn{9}{|l|}{ Stage } \\
\hline & I-II & 38 & 10 & $0.0020^{*}$ & 9 & $0.0072^{*}$ & 5 & $0.0026^{*}$ \\
\hline & III-IV & 86 & 49 & & 40 & & 32 & \\
\hline \multicolumn{9}{|c|}{$\begin{array}{l}\text { Histologic } \\
\text { al grade }\end{array}$} \\
\hline & $\begin{array}{l}\text { Keratinizing/ } \\
\text { Non-keratinizi } \\
\text { ng }\end{array}$ & 47 & 20 & 0.1980 & 17 & 0.2753 & 15 & 0.9521 \\
\hline & $\begin{array}{l}\text { Undifferentiat } \\
\text { ed }\end{array}$ & 77 & 39 & & 32 & & 22 & \\
\hline \multicolumn{9}{|l|}{$\begin{array}{l}\text { TRPA1 } \\
\text { level }\end{array}$} \\
\hline & $\begin{array}{l}\text { Low }(\text { H-score } \\
<\text { median) }\end{array}$ & 62 & 20 & $0.0003^{*}$ & 16 & $0.0005^{*}$ & 12 & $0.0026^{*}$ \\
\hline & $\begin{array}{l}\text { High (H-score } \\
\geq \text { median) }\end{array}$ & 62 & 39 & & 33 & & 25 & \\
\hline
\end{tabular}

*, Statistically significant; DSS, Disease-Specific Survival; DMeFS, Distal Metastasis-Free Survival; LRFS, local recurrence-free survival.
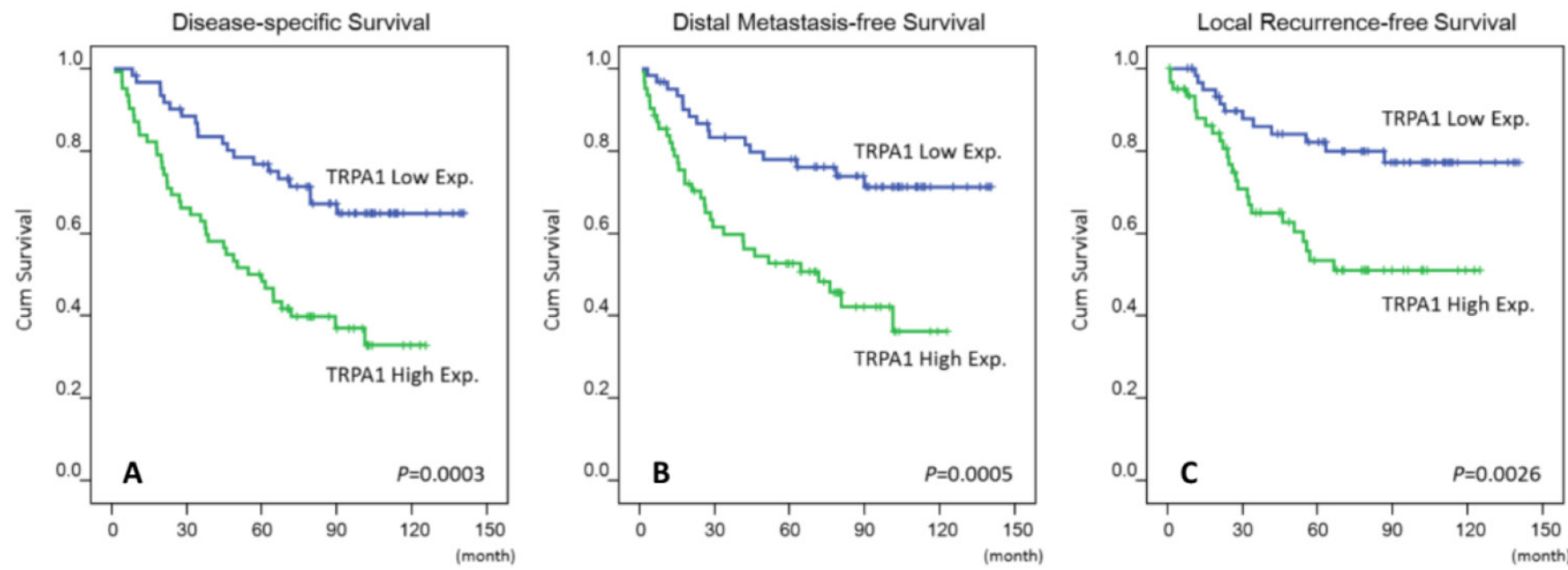

Figure 3. Kaplan-Meier estimator plotted the prognostic denotation of the TRPAI protein levels for disease-specific, distal metastasis-free and local recurrence-free survivals (A-C). 
Table 4. Multivariate survival analysis.

\begin{tabular}{|c|c|c|c|c|c|c|c|c|c|c|}
\hline \multirow[t]{2}{*}{ Parameter } & \multirow[t]{2}{*}{ Category } & \multicolumn{3}{|c|}{ DSS } & \multicolumn{3}{|c|}{ DMeFS } & \multicolumn{3}{|c|}{ LRFS } \\
\hline & & HR & $95 \% \mathrm{CI}$ & $P$-value & HR & $95 \% \mathrm{CI}$ & $P$-value & HR & $95 \% \mathrm{CI}$ & $P$-value \\
\hline \multicolumn{11}{|l|}{ Stage } \\
\hline & I-II & 1 & - & $0.003^{*}$ & 1 & - & $0.034^{*}$ & 1 & - & $0.015^{*}$ \\
\hline & III-IV & 2.374 & $1.191-4.730$ & & 2.211 & $1.062-4.602$ & & 3.261 & $1.256-8.470$ & \\
\hline \multicolumn{11}{|l|}{ TRPA1 level } \\
\hline & Low & 1 & - & $0.014^{*}$ & 1 & - & $0.004^{*}$ & 1 & - & $0.017^{*}$ \\
\hline & High & 2.297 & $1.328-3.972$ & & 2.471 & $1.346-4.535$ & & 2.335 & $1.161-4.696$ & \\
\hline
\end{tabular}

The 'wasabi receptor', i.e., TRPA1, plays a crucial part in somatosensation across diverse evolutionary phyla, including vertebrates and invertebrates [10]. In mammalian pain pathway, the TRPA1 protein is mainly expressed on primary afferent neurons. At these locations, TRPA1 works as a sensor of exogenous and endogenous chemical stimulants, for example, allyl isothiocyanate (AITC), acrolein, and 4-hydroxynonenal [19-22]. In human small cell lung cancer (SCLC), TRPA1 mRNA levels were markedly upregulated in tumor specimens, compared to normal lung tissues and non-SCLC samples. In vitro treatment with AITC, a volatile toxic substance, on respiratory system-derived SCLC cell lines, caused an increment of the concentration of intracellular calcium. This effect could be hampered by a specific antagonist of TRPA1, AP18. Through SRC proto-oncogene, non-receptor tyrosine kinaseand calcium-dependent machineries, AITC and formalin activated mitogen-activated protein kinase 1/3 [MAPK1/3, also known as extracellular signal regulated kinase 1/2 (ERK1/2)] in SCLC-derived TRPA1-positive H146 cells. Particularly, knockdown of the TRPA1 gene seriously damaged anchorage-independent cell growth in H146 cells [23]. For the first time, we found that the upregulation of TRPA1 protein correlates with the development and progression of NPC, but whether TRPA1 plays similar roles in NPC-derived cells to those of SCLC-derived cells remains to be elucidated.

Significantly increased hazard ratios of advanced AJCC stage (III-IV) and disease-specific, distal metastasis-free and local recurrence-free survivals in NPC patients were identified, similar to another investigation [24] and our previous studies $[16,25,26]$. Further, substantial correlations between TRPA1 protein levels and primary tumor in addition to the AJCC stage, reinforced that upregulation of TRPA1 protein may be responsible for NPC progression. Indeed, the intestinal inflammation associated with colon cancer is a well-known situation [27]. In mice, activation and sensitization of TRPA1 induced and maintained colitis [28]. Moreover, the TRPA1 mRNA level was upregulated in inflamed gut [29]. Accordingly, TRPA1 may serve as a high priority target via regulation of inflammation in the treatment of NPC.

Our study further indicated that the level of TRPA1 protein in biopsy specimens may anticipate a response to the traditional treatment on NPCs, as concurrent radiochemotherapy has no beneficial effects for quite a few NPC patients. In 4 years, about $30 \%$ to $40 \%$ of patients acquire metastatic properties. When metastasis arises, the prospect is extremely unfavorable [30]. It has been identified that extracellular molecules released by injured or inflammatory tissues regulate TRP channel members, including TRPV1, TRPV2, TRPV3, TRPV4, TRPM8 as well as TRPA1 [31]. Cell proliferation, invasion, migration and angiogenesis deeply implicate in tumor development [32]. These activities are partially regulated by calcium homeostasis including TRP cation channels [33-37]. By aiming at calcium homeostasis in cancer therapy, carboxyamidotriazole (CAI) was one pioneer drug in clinical trial [38]. CAI is a synthetic blocker of calcium influx and it displayed anti-angiogenic, -proliferative and -metastatic abilities in vitro. Nevertheless, treatment with CAI alone was not able to improve survival or quality of life in clinical trials in renal cell carcinoma [39], glioblastoma multiforme [40] and non-small cell lung cancer [41]. On the other hand, a consolidation of CAI and paclitaxel (a standard chemotherapy agent that targets tubulin, inhibits the disassembly of microtubules and cell division) enhanced anti-tumor activity in ovarian cancer [42]. While NPC varies from other carcinomas in its etiology and probable therapeutic alternatives, the results from our study implied that TRPA1 may be a novel molecular target for chemotherapy.

In conclusion, we conducted analysis of expression profile and assessment of TRPA1 expression in a cohort of 124 NPC patients. The TRPA1 protein levels could be detected by immunohistochemistry in all cases, in spite of the H-score varies a lot individually. In addition to the higher primary tumor, TRPA1 upregulation is independently and negatively predictive disease-specific, distal metastasis-free and local recurrence-free survivals. Therefore, TRPA1 signifies 
one valuable prognostic marker in patients with NPC, especially those who were remedied with radiation therapy and adjuvant cisplatin chemotherapy.

\section{Abbreviations}

AITC: allyl isothiocyanate; AJCC: American Joint Committee on Cancer; CAI: carboxyamidotriazole; EBV: Epstein-Barr virus; ERK1/2: extracellular signal regulated kinase 1/2; GEO: Gene Expression Omnibus; MAPK1/3: mitogen-activated kinase 1/3; NPC: nasopharyngeal carcinoma; SCLC: small cell lung cancer; TRPA1: transient receptor protein cation channel subfamily A, member 1; WHO: World Health Organization.

\section{Acknowledgements}

\section{This work was supported by grants from Chang Gung Hospital (CMRPG8D0711 and CMRPG8D0712 to YT Wu and SL Yen, respectively) and MOST 104-2314-B-110-002 (Ministry of Science and Technology, Taiwan) to YL Shiue.}

\section{Conflict of interest}

\section{The authors declare no conflict of interest.}

\section{References}

1. Tsao SW, Yip YL, Tsang CM, Pang PS, Lau VM, Zhang G, et al. Etiological factors of nasopharyngeal carcinoma. Oral oncology. 2014; 50(5):330-338.

2. Lo KW, To KF and Huang DP. Focus on nasopharyngeal carcinoma. Cancer cell. 2004; 5(5):423-428.

3. Chang ET and Adami HO. The enigmatic epidemiology of nasopharyngeal carcinoma. Cancer epidemiology, biomarkers \& prevention : a publication of the American Association for Cancer Research, cosponsored by the American Society of Preventive Oncology. 2006; 15(10):1765-1777.

4. Jeyakumar A, Brickman TM, Jeyakumar A and Doerr T. Review of nasopharyngeal carcinoma. Ear, nose, \& throat journal. 2006; 85(3):168-170, 172-163, 184

5. Chen YP, Guo R, Liu N, Liu X, Mao YP, Tang LL, et al. Efficacy of the Additional Neoadjuvant Chemotherapy to Concurrent Chemoradiotherapy for Patients with Locoregionally Advanced Nasopharyngeal Carcinoma: a Bayesian Network Meta-analysis of Randomized Controlled Trials. Journal of Cancer. 2015; 6(9):883-892.

6. Liang $X X, \mathrm{Li} Q, \mathrm{Su} Z$, Lan $X W$, Ouyang PY, Mao YP, et al. Significant prognostic impact of chemoradiotherapy-induced hemoglobin decrease on treatment outcomes of nasopharyngeal carcinoma. Journal of Cancer. 2015; 6(6):502-510.

7. Shi S, Li X, You B, Shan Y, Cao X and You Y. High Expression of FGFR4 Enhances Tumor Growth and Metastasis in Nasopharyngeal Carcinoma. Journal of Cancer. 2015; 6(12):1245-1254.

8. Venkatachalam $K$ and Montell C. TRP channels. Annual review of biochemistry. 2007; 76:387-417.

9. Zheng J. Molecular mechanism of TRP channels. Comprehensive Physiology. 2013; 3(1):221-242.

10. Nilius B and Owsianik G. The transient receptor potential family of ion channels. Genome biology. 2011; 12(3):218.

11. Owsianik G, D'Hoedt D, Voets T and Nilius B. Structure-function relationship of the TRP channel superfamily. Reviews of physiology, biochemistry and pharmacology. 2006; 156:61-90.

12. Gaudet R. TRP channels entering the structural era. The Journal of physiology. 2008; 586(Pt 15):3565-3575

13. Latorre R, Zaelzer C and Brauchi S. Structure-functional intimacies of transient receptor potential channels. Quarterly reviews of biophysics. 2009; 42(3):201-246.

14. Liberati S, Morelli MB, Nabissi M, Santoni M and Santoni G. Oncogenic and anti-oncogenic effects of transient receptor potential channels. Current topics in medicinal chemistry. 2013; 13(3):344-366

15. Chen J, Luan Y, Yu R, Zhang Z, Zhang J and Wang W. Transient receptor potential (TRP) channels, promising potential diagnostic and therapeutic tools for cancer. Bioscience trends. 2014; 8(1):1-10.
16. Hsu HP, Li CF, Lee SW, Wu WR, Chen TJ, Chang KY, et al. Overexpression of stathmin 1 confers an independent prognostic indicator in nasopharyngeal carcinoma. Tumour biology : the journal of the International Society for Oncodevelopmental Biology and Medicine. 2014; 35(3):2619-2629.

17. Ding $X, H e ~ Z$, Shi $Y$, Wang $Q$ and Wang $Y$. Targeting TRPC6 channels in oesophageal carcinoma growth. Expert opinion on therapeutic targets. 2010; 14(5):513-527.

18. Ouadid-Ahidouch H, Dhennin-Duthille I, Gautier $M$, Sevestre $H$ and Ahidouch A. TRP channels: diagnostic markers and therapeutic targets for breast cancer? Trends in molecular medicine. 2013; 19(2):117-124.

19. Bandell M, Story GM, Hwang SW, Viswanath V, Eid SR, Petrus MJ, et al. Noxious cold ion channel TRPA1 is activated by pungent compounds and bradykinin. Neuron. 2004; 41(6):849-857.

20. Bautista DM, Jordt SE, Nikai T, Tsuruda PR, Read AJ, Poblete J, et al. TRPA1 mediates the inflammatory actions of environmental irritants and proalgesic agents. Cell. 2006; 124(6):1269-1282.

21. Jordt SE, Bautista DM, Chuang HH, McKemy DD, Zygmunt PM, Hogestatt ED, et al. Mustard oils and cannabinoids excite sensory nerve fibres through the TRP channel ANKTM1. Nature. 2004; 427(6971):260-265.

22. Trevisani M, Siemens J, Materazzi S, Bautista DM, Nassini R, Campi B, et al. 4-Hydroxynonenal, an endogenous aldehyde, causes pain and neurogenic inflammation through activation of the irritant receptor TRPA1. Proceedings of the National Academy of Sciences of the United States of America. 2007; 104(33):13519-13524.

23. Schaefer EA, Stohr S, Meister M, Aigner A, Gudermann T and Buech TR. Stimulation of the chemosensory TRPA1 cation channel by volatile toxic substances promotes cell survival of small cell lung cancer cells. Biochemical pharmacology. 2013; 85(3):426-438.

24. Lee AW, Tung SY, Chan AT, Chappell R, Fu YT, Lu TX, et al. A randomized trial on addition of concurrent-adjuvant chemotherapy and/or accelerated fractionation for locally-advanced nasopharyngeal carcinoma. Radiotherapy and oncology : journal of the European Society for Therapeutic Radiology and Oncology. 2011; 98(1):15-22.

25. Lee SW, Chen TJ, Lin LC, Li CF, Chen LT, Hsing CH, et al. Overexpression of thymidylate synthetase confers an independent prognostic indicator in nasopharyngeal carcinoma. Experimental and molecular pathology. 2013; 95(1):83-90.

26. Lee SW, Lin CY, Tian YF, Sun DP, Lin LC, Chen LT, et al. Overexpression of CDC28 protein kinase regulatory subunit $1 \mathrm{~B}$ confers an independent prognostic factor in nasopharyngeal carcinoma. APMIS : acta pathologica, microbiologica, et immunologica Scandinavica. 2014; 122(3):206-214.

27. Rubin DC, Shaker A and Levin MS. Chronic intestinal inflammation: inflammatory bowel disease and colitis-associated colon cancer. Frontiers in immunology. 2012; 3:107.

28. Engel MA, Leffler A, Niedermirtl F, Babes A, Zimmermann K, Filipovic MR, et al. TRPA1 and substance P mediate colitis in mice. Gastroenterology. 2011; 141(4):1346-1358.

29. Izzo AA, Capasso $R$, Aviello $G$, Borrelli $F$, Romano B, Piscitelli $F$, et al. Inhibitory effect of cannabichromene, a major non-psychotropic cannabinoid extracted from Cannabis sativa, on inflammation-induced hypermotility in mice. British journal of pharmacology. 2012; 166(4):1444-1460.

30. Le QT, Tate D, Koong A, Gibbs IC, Chang SD, Adler JR, et al. Improved local control with stereotactic radiosurgical boost in patients with nasopharyngeal carcinoma. International journal of radiation oncology, biology, physics. 2003; 56(4):1046-1054.

31. Vay L, Gu C and McNaughton PA. The thermo-TRP ion channel family: properties and therapeutic implications. British journal of pharmacology. 2012; 165(4):787-801.

32. Gautier M, Dhennin-Duthille I, Ay AS, Rybarczyk P, Korichneva I and Ouadid-Ahidouch H. New insights into pharmacological tools to TR(i)P cancer up. British journal of pharmacology. 2014; 171(10):2582-2592.

33. Nilius B. Transient receptor potential (TRP) cation channels: rewarding unique proteins. Bulletin et memoires de l'Academie royale de medecine de Belgique. 2007; 162(3-4):244-253.

34. Wu LJ, Sweet TB and Clapham DE. International Union of Basic and Clinical Pharmacology. LXXVI. Current progress in the mammalian TRP ion channel family. Pharmacological reviews. 2010; 62(3):381-404.

35. Prevarskaya N, Zhang L and Barritt G. TRP channels in cancer. Biochimica et biophysica acta. 2007; 1772(8):937-946.

36. Prevarskaya N, Skryma R and Shuba Y. Ion channels and the hallmarks of cancer. Trends in molecular medicine. 2010; 16(3):107-121.

37. Alexander SP, Benson HE, Faccenda E, Pawson AJ, Sharman JL, Catterall WA et al. The Concise Guide to PHARMACOLOGY 2013/14: ion channels. British journal of pharmacology. 2013; 170(8):1607-1651.

38. Kohn EC, Figg WD, Sarosy GA, Bauer KS, Davis PA, Soltis MJ, et al. Phase I trial of micronized formulation carboxyamidotriazole in patients with refractory solid tumors: pharmacokinetics, clinical outcome, and comparison of formulations. Journal of clinical oncology : official journal of the American Society of Clinical Oncology. 1997; 15(5):1985-1993.

39. Stadler WM, Rosner G, Small E, Hollis D, Rini B, Zaentz SD, et al. Successful implementation of the randomized discontinuation trial design: an application to the study of the putative antiangiogenic agent carboxyaminoimidazole in renal cell carcinoma--CALGB 69901. Journal of clinical oncology : official journal of the American Society of Clinical Oncology. 2005; 23(16):3726-3732. 
40. Mikkelsen T, Lush R, Grossman SA, Carson KA, Fisher JD, Alavi JB, et al. Phase II clinical and pharmacologic study of radiation therapy and carboxyamido-triazole (CAI) in adults with newly diagnosed glioblastoma multiforme. Investigational new drugs. 2007; 25(3):259-263.

41. Johnson EA, Marks RS, Mandrekar SJ, Hillman SL, Hauge MD, Bauman MD, et al. Phase III randomized, double-blind study of maintenance CAI or placebo in patients with advanced non-small cell lung cancer (NSCLC) after completion of initial therapy (NCCTG 97-24-51). Lung cancer (Amsterdam, Netherlands). 2008; 60(2):200-207.

42. Azad N, Perroy A, Gardner E, Imamura CK, Graves C, Sarosy GA, et al. A phase I study of paclitaxel and continuous daily CAI in patients with refractory solid tumors. Cancer biology \& therapy. 2009; 8(19):1800-1805. 